\section{FRI0373 SUBSTANCE P AND VASCULAR ENDOTHELIAL GROWTH FACTOR PRODUCTION BY PERIPHERAL BLOOD MONONUCLEAR CELLS DERIVING FROM PATIENTS WITH SYSTEMIC SCLEROSIS}

A. Corrado, E. Sanpaolo, R. Colia, A. Mele, F.P. Cantatore. Department of Medical and Surgical Sciences - Rheumatology Clinic, University of Foggia, Foggia, Italy

Background: Many cytokines may potentially play a relevant role in the pathogenesis of different aspects of SSc, particularly fibrosis and endothelial injury. Some data suggest that substance $\mathrm{P}(\mathrm{SP})$ may act in an autocrine/paracrine manner to regulate vasoconstriction and/or immunologic and inflammatory responses. VEGF and MCP1, which are produced by inflammatory cells, may be involved in the pathogenesis of vascular changes and accumulation of extracellular matrix in SSc.

Objectives: The aim of this study was to determine the expression of Substance P (SP), Vascular Endothelial Growth Factor (VEGF), Monocyte Chemoattractant Protein-1 (MCP-1) in SSc patients and their relationship with the main clinical manifestations of disease

Methods: 34 SSc patients fulfilling the 2013 ACR/EULAR criteria for SSc and classified according to Leroy as having limited cutaneous (ISSc) or diffuse cutaneous (dSSc) disease were recruited. 28 sex and age matched healthy subjects was used as control. For each recruited patient we analyzed the disease characteristic, including the extent of skin fibrosis, the pattern of internal organ involvement, the presence of digital ulcers (DU) and autoantibody profile. Peripheral Blood Mononuclear Cells (PBMCs) were isolated and cultured from both SSc patients and healthy controls. The expression of SP, VEGF, MCP1 was analyzed by real-time polymerase chain reaction (PCR) and their concentrations where measured in supernatants of cultured PBMCs using enzyme-linked immunosorbent assay (ELISA). The correlation between cytokine expression and clinical manifestations was evaluated

Results: The results showed that the mRNA and the protein levels of SP and VEGF were significantly higher in SSc patients compared to controls $(190,55 \pm 59,1 \mathrm{pg} / \mathrm{mL}$ vs $128,85 \pm 69,8 \mathrm{pg} / \mathrm{mL}$ and $422,31 \pm 149,4 \mathrm{pg} / \mathrm{mL}$ vs $309,25 \mathrm{pg} / \mathrm{mL}$ respectively, $\mathrm{p}<0,05)$; further, SP and VEGF levels were higher in dSSc compared to ISSc and correlated to disease duration. No difference was detected in MCP1 synthesis and expression between SSc subjects and healthy controls $(507,17 \pm 153,9 \mathrm{pg} / \mathrm{mL}$ vs $481,2 \pm 118,2 \mathrm{pg} / \mathrm{mL}$ ) even if in dSSc patients a slight increase was observed. VEGF production negatively correlated with the prevalence of DU. No correlation between cytokines production and heart, lung and gastrointestinal involvement and autoantibody profile was found.

Conclusions: The preliminary data of this study show that SP and VEGF are over-expressed in PBMCs of SSc subjects and are related to the extent of skin involvement, whereas only VEGF production negatively correlates with the prevalence of DU. The exact role played by SP and VEGF in the pathogenesis of vascular injury and fibrosis in SSc and their relationship with the immune abnormalities observed in this disease is not completely clear and the available data are contrasting. Additional in vitro and in vivo studies are needed to better understand the role of SP and VEGF in the pathogenesis of SSc.

Disclosure of Interest: None declared

DOI: 10.1136/annrheumdis-2017-eular.6587

\section{FRI0374 BONE MINERAL DENSITY AND TRABECULAR BONE SCORE} IN TWO SUBSETS OF SYSTEMIC SCLEROSIS PATIENTS

A. Corrado, A. Mele, A. Trotta, R. Colia, S. Di Bello, F.P. Cantatore. Department of Medical and Surgical Sciences - Rheumatology Clinic, University of Foggia, Foggia, Italy

Background: Data concerning the relationship between Systemic Sclerosis (SSc) and alterations of Bone Mineral Density (BMD) are very conflicting. The established standard for measuring BMD is Dual X-ray Absorptiometry (DXA), but it does not provide any informations about the bone microarchitecture, which is a parameter very difficult to evaluate in clinical practice but it is essential to define bone strength. The Trabecular Bone Score (TBS) is a new structural parameter that can be obtained by DXA scanning and it is related to bone microarchitecture and provides data on bone quality irrespective of bone density.

Objectives: The aim of this study is to evaluate the differences in BMD and TBS values in two subsets of patients with SSc (limited and diffuse cutaneous disease - ISSc and dSSc) and to analyse their possible relationship with the parameters of body mass composition and phospho-calcium metabolism.

Methods: 64 post-menopausal patients classified according to Leroy as limited cutaneous (ISSc) or diffuse cutaneous (dSSc) SSc, were recruited. The following clinical parameters were evaluated: internal organ involvement, degree of skin involvement (Rodnan Skin Score), bone mass index (BMI). For each recruited patient, spine and hip BMD, TBS and body mass composition (lean and fat mass, total bone mineral content - BMC) were assessed by DXA. Serum calcium, phosphorus, alkaline phosphatase, intact parathyroid hormone (PTH) and 25-hydroxyvitamin D (25 OHD) were also measured.

Results: BMD at femoral neck and spine was significantly higher in ISSc compared to dSSc patients $(p<0,04)$. In ISSc subjects also BMC was significantly higher compared to dSSc ones $(p<0,01)$ but, interestingly the TBS was significantly lower $(p<0,03)$. ISSc patients presented a higher values of fat mass compared to dSSc $(\mathrm{p}<0,05)$, with a slightly higher BMI (which did not reach statistical significance). $25 \mathrm{OH}$ serum levels were higher in ISSc group, whereas no differences between ISSc and dSSc in serum calcium, phosphorus, alkaline phosphatase were observed.

Conclusions: The presented preliminary data show that in SSc patients with limited cutaneous disease, the BMD values are higher compared to patient with diffuse cutaneous disease; nevertheless in this subset (ISSc) bone quality seem to be reduced, as TBS values are significantly lower. Higher BMC values and serum $25 \mathrm{OHD}$ levels observed in ISSc patients could be among the factors that contribute to increasing BMD in this clinical subset. Further analysis should be performed to identify the possible causes of the reduced bone quality in spite of higher BMD in ISSc patients, such as the study of serum adipokines, considering that the presented data show a great fat mass in patients with limited cutaneous disease.

References:

[1] Harvey NC. et al. Trabecular bone score (TBS) as a new complementary approach for osteoporosis evaluation in clinical practice. Bone. 2015 Sep;78:216-24

[2] Bousson V. et al. Trabecular Bone Score: Where are we now? Joint Bone Spine. 2015 Oct;82(5):320-5.

[3] Corrado A et al. Relationship between Body Mass Composition, Bone Mineral Density, Skin Fibrosis and 25(OH) Vitamin D Serum Levels in Systemic Sclerosis. PLoS One. 2015 Sep 16;10(9).

Disclosure of Interest: None declared

DOI: 10.1136/annrheumdis-2017-eular.5207

\section{FRI0375 REAL-LIFE TREATMENT STRATEGIES FOR SYSTEMIC SCLEROSIS ACCORDING TO EXPERTS}

A. Fernández-Codina ${ }^{1}$, K.M. Walker ${ }^{2}$, J.E. Pope ${ }^{3}$ on behalf of the participating members of the Scleroderma Clinical Trials Consortium (SCTC) and Canadian Scleroderma Research Group (CSRG). ${ }^{1}$ Systemic autoimmune diseases unit, Hospital Universitari Vall d'Hebron, Barcelona, Spain; ${ }^{2}$ Rheumatology, The Ottawa Hospital, Ottawa; ${ }^{3}$ Rheumatology, University of Western Ontario, London, Canada

Background: Second line treatment options for Systemic Sclerosis (SSc) are limited, and scarce data are available for choosing the order of treatment.

Objectives: The aim of this study is to update the SSc treatment algorithms obtained in $2012^{1}$, based on SSc experts' daily practice.

Methods: An initial survey was designed based on the 2012 algorithms. The survey asked experts whether they agreed with the 2012 algorithms or not, and which changes should be considered. The questionnaire was completed by 62 of 168 surveyed (67\% response) between August and October 2016.

Results: For scleroderma renal crisis (SRC), there was $65 \%$ to $69 \%$ agreement with the previous algorithms (1st line angiotensin converting enzyme inhibitors [ACEI], 2nd and 3rd adding: calcium channel blockers [CCB] or angiotensin receptor blockers [ARB], and 4th alpha-blocker). In mild pulmonary arterial hypertension (PAH), only $45 \%$ of the experts agreed with the old algorithm. The majority suggested first phosphodiesterase 5 inhibitors (PDE5i) or endotelin receptor antagonists (ERA) plus PDE5i, then prostanoids. In severe $\mathrm{PAH}, 65 \%$ agreed with the preexistent scheme (1st prostanoids, 2nd ERA plus PDE5i, 3rd ERA plus prostanoids). For mild Raynaud's phenomenon (RP) $66 \%$ agreed with the previous algorithm (1st CCB, 2nd adding PDE5i, 3rd ARB or switching to another $\mathrm{CCB}$, 4th prostanoids). Regarding severe RP, $52 \%$ agreed with previous (1st CCB, 2nd adding PDE5i, 3rd ERA, 4th prostanoids). Conversely, 60\% of the experts did not agree with the prior active digital ulcer (DU) treatment, suggesting 1st CCB, 2nd PDE5i, 3rd prostanoids. For interstitial lung disease (ILD), for induction only $24 \%$ agreed with the older proposal. Experts suggested 1st mofetil mycophenolate (MMF), 2nd intravenous (IV) cyclophosphamide (CYP), 3rd Rituximab. There was a $65 \%$ agreement on ILD maintenance (1st MMF, 2nd azathioprine [AZA], 3rd IV CYP, 4th oral CYP). For skin involvement, agreement for patients with a modified Rodnan skin score (mRSS) of 10 was 57\% (1st methotrexate [MTX]), 2nd MMF); if the mRSS was 24, 32\% suggested 1st MMF, 2nd MTX; and mRSS 32, 36\% chose 1st MTX, 2nd MMF, 3rd IV CYP, 4th autologous stem cell transplantation (ASCT). In inflammatory arthritis $45 \%$ agreed with the previous algorithm, whereas others suggested 1st MTX, 2nd low dose steroids, 3rd hydroxychloroquine, 4th rituximab or tocilizumab.

Conclusions: There remains some disagreement for 2 nd line treatment of SSc. Combination of PDE5 $\mathrm{i}$ and ERA are prescribed now in mild $P A H$ treatment. Prostanoids have been incorporated as 3rd line agents in active $D U$ treatment. MMF is the new 1 st line treatment for ILD induction and rituximab the 3rd. IV CYA and ASCT were recommended as 3rd and 4th line treatments in patients with severe skin involvement. Rituximab and tocilizumab have been incorporated into inflammatory arthritis treatment. This can guide therapy in SSc.

\section{References:}

[1] Walker KM, Pope J. Treatment of systemic sclerosis complications: what to use when first-line treatment fails-a consensus of systemic sclerosis experts. Semin Arthritis Rheum 2012;42(1):42-55

Disclosure of Interest: None declared

DOI: 10.1136/annrheumdis-2017-eular.1574 